University of Wollongong

Research Online

Faculty of Social Sciences - Papers (Archive) Faculty of Arts, Social Sciences \& Humanities

2019

Data-driven derivation of natural EEG frequency components: An optimised example assessing resting EEG in healthy ageing

Robert J. Barry

University of Wollongong, rbarry@uow.edu.au

Frances M. De Blasio

University of Wollongong, francesd@uow.edu.au

Diana Karamacoska

University of Wollongong, dk744@uowmail.edu.au

Follow this and additional works at: https://ro.uow.edu.au/sspapers

Part of the Education Commons, and the Social and Behavioral Sciences Commons

Research Online is the open access institutional repository for the University of Wollongong. For further information contact the UOW Library: research-pubs@uow.edu.au 


\title{
Data-driven derivation of natural EEG frequency components: An optimised example assessing resting EEG in healthy ageing
}

\author{
Abstract \\ Background: The majority of electroencephalographic (EEG) investigations in normal ageing have \\ determined EEG spectra from epochs recorded in the eyes-closed (EC) and/or eyes-open (EO) resting \\ states, and summed amplitudes or power estimates within somewhat-arbitrary and/or inconsistently \\ defined traditional frequency band limits. New method: Natural frequency components were sought using \\ a data-driven frequency Principal Components Analysis (f-PCA) approach, optimised to reduce between- \\ condition and between-group misallocation of variance. Frequency component correspondence was \\ screened using the Congruence Coefficient and topographic correlations for potential matches on \\ Condition and/or Group. The amplitudes of corresponding natural components were then explored as a \\ function of these independent variables. Results: Separate f-PCAs with Young and Older adults' EC and \\ EO data each yielded between six and nine components that peaked across the traditional delta to beta \\ band ranges. Across these, two components were matched on Group and Condition, while a further six \\ were matched on Condition (within-groups), and four on Group (within-conditions). Comparison with \\ Existing Methods: Multiple frequency components were found within the traditional bands, and provided a \\ wider perspective in terms of additional natural component details. In addition to novel insights, the well- \\ documented age-related spectral reductions were seen in the common delta component, and in one EC \\ (but no EO) alpha component. Conclusions: This combination of optimised f-PCA approach and \\ component screening procedure has wide potential in the EEG field beyond the ageing topic explored \\ here, being applicable across an extensive range of studies using EEG oscillations to explore aspects of \\ cognitive processing and individual differences.

\section{Disciplines \\ Education | Social and Behavioral Sciences}

\section{Publication Details} \\ Barry, R. J., De Blasio, F. M. \& Karamacoska, D. (2019). Data-driven derivation of natural EEG frequency \\ components: An optimised example assessing resting EEG in healthy ageing. Journal of Neuroscience \\ Methods, 321 1-11.
}


Data-driven derivation of natural EEG frequency components: An optimised example assessing resting EEG in healthy ageing

Robert J. Barry*, Frances M. De Blasio, Diana Karamacoska

Brain \& Behaviour Research Institute and School of Psychology, University of Wollongong, Wollongong, NSW 2522, Australia

*Corresponding author:

Email: rbarry@uow.edu.au

Phone/fax: +612 42214421

Address: School of Psychology,

University of Wollongong,

Northfields Avenue,

Wollongong NSW 2522,

Australia. 


\section{Highlights}

*Frequency PCA was used to decompose resting EEG spectra in Young and Older adults *Optimised protocol minimises misallocation of variance, improves component structure *Component matches across group and/or condition confirmed via Congruence Coefficient *Data-driven frequency components peaked across traditional bands; most were in alpha *Natural frequency components provide insight beyond traditional band analyses 


\section{Abstract}

Background: The majority of electroencephalographic (EEG) investigations in normal ageing have determined EEG spectra from epochs recorded in the eyes-closed (EC) and/or eyes-open (EO) resting states, and summed amplitudes or power estimates within somewhatarbitrary and/or inconsistently defined traditional frequency band limits.

New Method: Natural frequency components were sought using a data-driven frequency Principal Components Analysis (f-PCA) approach, optimised to reduce between-condition and between-group misallocation of variance. Frequency component correspondence was screened using the Congruence Coefficient and topographic correlations for potential matches on Condition and/or Group. The amplitudes of corresponding natural components were then explored as a function of these independent variables.

Results: Separate f-PCAs with Young and Older adults' EC and EO data each yielded between six and nine components that peaked across the traditional delta to beta band ranges. Across these, two components were matched on Group and Condition, while a further six were matched on Condition (within-groups), and four on Group (within-conditions). Comparison with Existing Methods: Multiple frequency components were found within the traditional bands, and provided a wider perspective in terms of additional natural component details. In addition to novel insights, the well-documented age-related spectral reductions were seen in the common delta component, and in one EC (but no EO) alpha component. Conclusions: This combination of optimised f-PCA approach and component screening procedure has wide potential in the EEG field beyond the ageing topic explored here, being applicable across an extensive range of studies using EEG oscillations to explore aspects of cognitive processing and individual differences.

Keywords: resting EEG, eyes-closed vs. eyes open, natural EEG components, Frequency Principal Components Analysis (f-PCA), normal ageing, misallocation of variance 


\section{Introduction}

\subsection{Brain oscillations and healthy ageing}

Changes in resting electroencephalographic (EEG) activity across the lifespan have long been of interest in neuroscience. Most of the research in this field has assessed the traditional delta ( $\sim 0.5$ to $3.5 \mathrm{~Hz})$, theta $(\sim 4.0$ to $7.5 \mathrm{~Hz})$, alpha $(\sim 8.0$ to $13.0 \mathrm{~Hz})$, and beta ( 13.5 to 24.0-30.0 Hz) bands, and reported data from eyes-closed (EC) resting conditions. The general consensus has been that delta activity decreases with increasing age (e.g., Babiloni et al., 2006; Barry and De Blasio, 2017; Breslau et al., 1989; Duffy et al., 1984; Gaál et al., 2010; Giaquinto and Nolfe, 1986; Polich, 1997; Sleimen-Malkoun et al., 2015; Vysata et al., 2012). Theta activity also shows an age-related decrease (e.g., Barry and De Blasio, 2017; Breslau et al., 1989; Cummins and Finnigan, 2007; Duffy et al., 1984; Polich, 1997; Sleimen-Malkoun et al., 2015; Vysata et al., 2012), and a similar finding occurs in the alpha band (e.g., Babiloni et al., 2006; Barry and De Blasio, 2017; Breslau et al., 1989; Fan et al., 2014; Polich, 1997; Sleimen-Malkoun et al., 2015; Vysata et al., 2012). The higher frequency beta results, however, have been more varied, with reports of both increases with age (e.g., Barry and De Blasio, 2017; Duffy et al., 1984; Fan et al., 2014; Giaquinto and Nolfe, 1986; Marciani et al., 1994; Wang et al., 2016) and decreases with age (e.g., Breslau et al., 1989; Vysata et al., 2012). Moreover, comparable age effects have also generally been reported in each of these bands during eyes-open (EO) resting (e.g., Barry and De Blasio, 2017; Vlahou et al., 2014; Wang et al., 2016).

The early ageing EEG literature largely ignored scalp distribution, often using few electrodes, with later reports sometimes grouping electrodes into broad regions (e.g., frontal/parietal). Overall, it appears that delta, theta, and alpha activity may become less midline dominant with increasing age (Babiloni et al., 2006; Breslau et al., 1989), although alpha seems to remain occipitally-focussed (Giaquinto and Nolfe, 1986); and beta may become more right-hemispheric (Fan et al., 2014). Barry and De Blasio (2017) found that increasing age reduced delta and theta more in midline and posterior regions, reduced alpha 
more in the right hemisphere and posterior regions, and increased beta more in left hemisphere, left parietal, and frontocentral regions; generally confirming previous studies, apart from that in the beta band.

Changes between EC and EO resting conditions have not been widely explored in healthy ageing. Most studies have focussed on alpha reactivity, with decreased EO reductions reported with increasing age (Duffy et al., 1984; Klimesch, 1999; Könönen and Partanen, 1993). Barry and De Blasio (2017) confirmed this age-related reduction in alpha reactivity, primarily in alpha's core posterior topography, and reported additional focal decreases in reactivity in each of the remaining traditional bands.

\subsection{New methodology}

It is unclear how, and to what extent, these findings have been affected or constrained by the traditional band ranges employed in these studies, such as $0.5-3.5 \mathrm{~Hz}$ for delta, $4.0-$ 7.5 Hz for theta, $8.0-13.0 \mathrm{~Hz}$ for alpha, and $13.5-24.0 \mathrm{~Hz}$ for beta. These were the limits used in Barry and De Blasio (2017), and in the absence of agreed band ranges, many studies have employed variations on these values, and some have even subdivided the higher frequency bands (i.e., alpha and/or beta) into low and high band ranges. Rather than rest on these somewhat arbitrary values, here we sought to explore the frequency components naturally occurring in these young and older participants. To that end we applied a relatively-new and promising, but not yet widely adopted, technique to decompose the EEG frequency spectrum using Frequency Principal Components Analyses (f-PCA).

Temporal PCA (t-PCA) has been increasingly used to partition the voltage waveform over time of the event related potential (ERP) into its components, such as the well-known N100 and P300, as well as dividing these further into their subcomponents. f-PCA is an analogous decomposition of the EEG voltage distribution over frequency, using the same mathematics as standard t-PCA (Barry and De Blasio, 2018; Barry et al., submitted; Debener et al., 2000; Kayser et al., 2000, 2014; Tenke and Kayser, 2005; Tenke et al., 2015, 2017a, 
2017b). This implementation with spectral data was first advanced by Debener et al. (2000) and Kayser et al. (2000), with the inherent advantage of providing a data-driven (cf. somewhat inconsistent and/or arbitrary) approach across the traditional bands.

While the use of this innovative quantification technique appears to be gathering momentum, some two decades after its introduction, its execution has not generally been optimal. For example, although many published EC/EO f-PCA studies to date have followed the methodology advocated by Kayser et al. (2000) - using the covariance matrix as input, with extraction and rotation of all components - most have submitted their data to a combined (across condition) PCA, and applied Varimax rotation. PCAs on combined datasets have been shown (in the analogous t-PCA ERP studies) to suffer from misallocation of variance between conditions (Barry et al., 2016). In essence, a combined PCA across conditions mixes variance from the components dominant in the separate conditions. Separate PCAs for each condition are required to reduce this problem, as indicated in ERP data (Barry et al., 2016), with recent confirmation similarly indicated in f-PCA studies of resting EEG (Barry et al., submitted; Karamcoska et al., submitted). Although Varimax rotation is recommended in t-PCA (Kayser et al., 2000; Kayser and Tenke, 2003), its application in f-PCA has been shown recently to be inferior compared with Promax rotation. Barry and De Blasio (2018) compared unrestricted Varimax and Promax rotation of f-PCA components, and the latter was associated with fewer instances of negative scaled factor loadings, a simpler component structure post-rotation, and component inter-correlations that indicated substantial inter-dependence ( 12\% shared variance), suggesting that forced component orthogonality (as in Varimax rotation) is unwise; we provide an example of this in Supplementary Material (section S1). Based on our prior work, we therefore regard this combination of f-PCAs on separate datasets, coupled with unrestricted extraction and Promax rotation, to be optimal in separating natural EEG components.

One issue that may have hindered the use of separate PCAs between conditions and/or groups is how to handle the large number of components produced, which increase with the 
number of separate datasets. Statistical comparisons can only be made between corresponding components, and this requires screening between condition- and/or groupoutputs to identify/confirm correspondence. Tucker's (1951) Congruence Coefficient $\left(r_{c}\right.$; also see Lorenzo-Seva and ten Berge, 2006), is a variant of correlation between unscaled factor loadings that can serve this purpose. This measure is independent of topography, and in its f-PCA implementation reflects similarities in the component waveform over frequency, and spectral amplitude. Topographic consistency can then be examined via correlation over the paired scalp sites, which provides a simple indication of the topographic correspondence between spectral components. This screening methodology has proved useful in t-PCA applications (e.g., Barry et al., 2016), and has been successfully applied in recent f-PCA investigations (e.g., Barry et al., submitted).

\subsection{Revisiting healthy ageing with new tools}

This study re-examined the frequency spectra reported in Barry and De Blasio (2017). In that investigation, resting EC and EO EEG data for samples of young and older adults were quantified using the traditional band approach in order to assess spectral changes in healthy ageing. We now implement f-PCA, utilising the refined protocol and screening tools outlined in Section 1.2, to quantify the natural frequency components inherent in these data, again assessing them from a healthy ageing perspective in order to demonstrate the advantages of this quantification technique. Based on the limited previous f-PCA studies in young adults (e.g., Barry and De Blasio, 2018; Barry et al., submitted; Debener et al., 2000; Kayser et al., 2000), we expected to find several frequency components in each of the delta and alpha ranges, with less well-defined components in theta, and perhaps one or two beta components. A broadly-comparable set of frequency components was anticipated in the older adults. We also expected to uncover frequency components that differed with condition (EO vs. EC) and/or age (young vs. older adults), although the nature of these differences was difficult to predict. That is, there is no logical necessity that different components within a 
broad (traditional) frequency range should shift together - for example, if the broad alpha band amplitudes decrease with age, do the multiple alpha components peaking within this frequency range necessarily change together?

Despite the novelty of this investigation, tentative hypotheses were derived from the traditional band literature sketched above. We generally expected reductions in component amplitudes (in all bands) from EC to EO, as well as age-related amplitude reductions in delta, theta, and alpha components, and age-related amplitude increases in beta components; all with some topographic differences. Shifts in peak alpha frequency could also be anticipated, as has been reported in the traditional ageing literature (e.g., Barry and De Blasio, 2017; Klimesch, 1999), although peak frequencies in the other bands have not been systematically explored. Details in relation to EC/EO changes in components are somewhat unclear due to prior studies having generally employed combined PCAs across condition cf. separate PCAs. Hence the present study, applying our optimised f-PCA quantification technique, is expected to yield novel insights into the frequency structure of the EEG from healthy young and older participants, and how this changes from EC to EO conditions.

\section{Methods}

This study implemented improved quantification methodology (i.e., f-PCA cf. traditional band quantification) in the re-assessment of the EEG data presented in Barry and De Blasio (2017). Accordingly, the participant characteristics and pre-PCA data quantification protocol are briefly summarised.

\subsection{Participants}

A convenience sample of twenty independent-living retirees (15 female) constituted the older adult group; they had a mean age of 68.2 years $(S D=4.5$ years $)$ and received $\mathrm{A} \$ 40$ for their participation. Twenty gender-matched university students constituted the young adult group; they had a mean age of 20.4 years $(S D=1.6$ years) and received course credit for their 
participation. All participants were screened for self-reported head injury, neurological disorders, psychiatric conditions and learning disability. The older group were additionally screened for cognitive impairment and reported their current medication intake. Abstinence from caffeine and other psychoactive substances was requested for $\geq 2.5 \mathrm{~h}$ prior to testing, and written informed consent was obtained in line with institutional ethics committee requirements.

\subsection{Electrophysiological recording}

Nineteen channels of scalp EEG from the standard 10-20 system and A2 were recorded relative to A1, and subsequently re-referenced to digitally averaged ears. Vertical and horizontal EOGs were recorded and an initial calibration task was used to allow removal of ocular artefact via a regression technique (Croft and Barry, 2000). Three minutes each of EC and EO data were recorded $\mathrm{DC}-30 \mathrm{~Hz}$ at a sampling rate of 1,000 Hz. The EO condition employed a fixation point to reduce eye movements.

\subsection{Data extraction and processing}

Sequential 2 second epochs were extracted from the EOG-corrected EEG data in each condition (EC, EO), baselined across their duration (i.e., DC corrected), and those with extreme amplitudes (exceeding $\pm 100 \mu \mathrm{V}$ ) were automatically identified and discarded. For each artefact-free epoch, data were separately subjected to a $10 \%$ Hanning window, and a Discrete Fourier Transformation (DFT) was used to derive the single-sided amplitude spectra $^{1}$; amplitudes were then corrected for the reduction by the window. Averaging spectral amplitudes across the accepted epochs falling within each 1 min segment (cf. the

${ }^{1}$ Note that double-sided spectra were assessed in Barry and De Blasio (2017), thus the present spectral amplitudes are consistently (i.e., for each Group and Condition) doubled in amplitude. 
accepted epochs across the 3 min recording as in Barry and De Blasio, 2017) yielded three (independent) mean spectra for each participant in each condition (EC, EO), ranging from DC $-30 \mathrm{~Hz}$ with a resolution of $0.5 \mathrm{~Hz}$. These three replicates increase the database for the fPCA and enhance output stability. A separate f-PCA was carried out for each condition in each group. Each of these contained 61 variables (amplitudes of the frequencies from DC-30 $\mathrm{Hz})$ and 1,140 cases (20 participants $\times 3$ spectra $\times 19$ electrodes $)$ for a case:variable ratio of 18.7. Following Barry and De Blasio (2018) and Barry et al. (submitted), the f-PCA used the covariance matrix with Kaiser normalisation, followed by unrestricted component rotation using Promax, in Dien's (2010) PCA toolkit (v. 2.23). Only components accounting for $\geq 2 \%$ variance were considered for investigation.

\subsection{Statistical analyses}

The numbers of artefact-free epochs in each 1 min segment for each condition and group were submitted to a three-way mixed MANOVA over Group (Young [Y], Older [O]), Condition (EC, EO), and Segment $(1,2,3)$, with repeated measures on the last two factors.

Screening of comparable components from different f-PCAs considered spectral consistency via the Congruence Coefficient ( $r_{c}$; Tucker, 1951), a variant of correlation between corresponding unscaled factor loadings. This is evaluated using a rule of thumb: $r_{c}$ $>.95$ indicates equality, and $.85 \leq r_{c} \leq .94$ indicates fair similarity (Lorenzo-Seva and ten Berge, 2006). As the Congruence Coefficient is independent of topography, topographic consistency was examined via correlations over the paired scalp sites. This correlation was expected to be positive, so its significance was tested with a one-tailed test.

In order to statistically compare corresponding frequency components, mean amplitudes were derived from a cluster of (at least three) peak topographic sites so as to avoid/minimise the influence of noise at a single site. The peak topography clusters were derived via visual inspection of the grand mean component topographic headmaps, and care was taken to ensure the consistency of these regions both within and between analyses (i.e., 
the clusters captured the peak topography in each component being compared). Frequency components that appeared from the initial screenings to be robust across Condition and Group were submitted to Analysis 1, a two-way mixed MANOVA, with the repeatedmeasures factor of Condition (EC, EO), and between factor of Group (Y, O). Frequency components that appeared to be robust across Condition (within-Group) were submitted to Analysis 2, a repeated-measures MANOVA over Condition (EC, EO). Frequency components that appeared from the screenings to be robust across Group (within-Condition) were submitted to Analysis 3, a one-way MANOVA with a between factor of Group (Y, O). As all contrasts were planned and did not exceed the degrees of freedom for effect, no Bonferroni-type adjustment of $\alpha$ levels was required (Tabachnick and Fiddell, 2013). $F$-tests involving Group have $d f=(1,38)$; single-group $F$-tests have $d f=(1,19)$.

\section{Results}

\subsection{EEG spectra}

Of the possible 30 epochs per Segment, few were lost to artefact: the Young group averaged $28.6( \pm 1.6)$ to $28.9( \pm 1.6)$ artefact-free epochs in each 1 min average for EC, and $29.3( \pm 1.7)$ to $29.6( \pm 1.4)$ epochs in EO. Corresponding epoch numbers for the Older group were $29.8( \pm 0.9)$ to $30.0( \pm 0.0)$ for EC, and $28.7( \pm 4.1)$ to $29.6( \pm 1.0)$ for EO. These epoch numbers did not differ with Condition, Segment, or Group, but a Group $\times$ Condition interaction $\left(F=7.20, p=.011, \eta_{\mathrm{p}}^{2}=.16\right)$ supported the observation that the Young group had fewer artefacts in EO than EC, while the Older group had fewer artefacts in EC than EO. Apart from that observation, the six mean EEG spectra examined here are based on comparable data across Group and Condition. Fig. 1 shows the EC and EO spectra for the Young and Older groups at the major midline sites and the occipital hemispheric mean. The traditional band ranges are indicated on the EO spectra (right panel) at $\mathrm{Cz}$. There is a major spectral peak in the low delta range, followed by the usual decrease with increasing frequency. This is interrupted by the alpha peak around 9-10 Hz, most dominant at Pz and 
$\mathrm{O} 1 / \mathrm{O} 2$ in EC (left panel). Alpha is reduced in EO, and the Older group appears to have lower amplitudes than the Young group across the frequency range, except for that in beta.
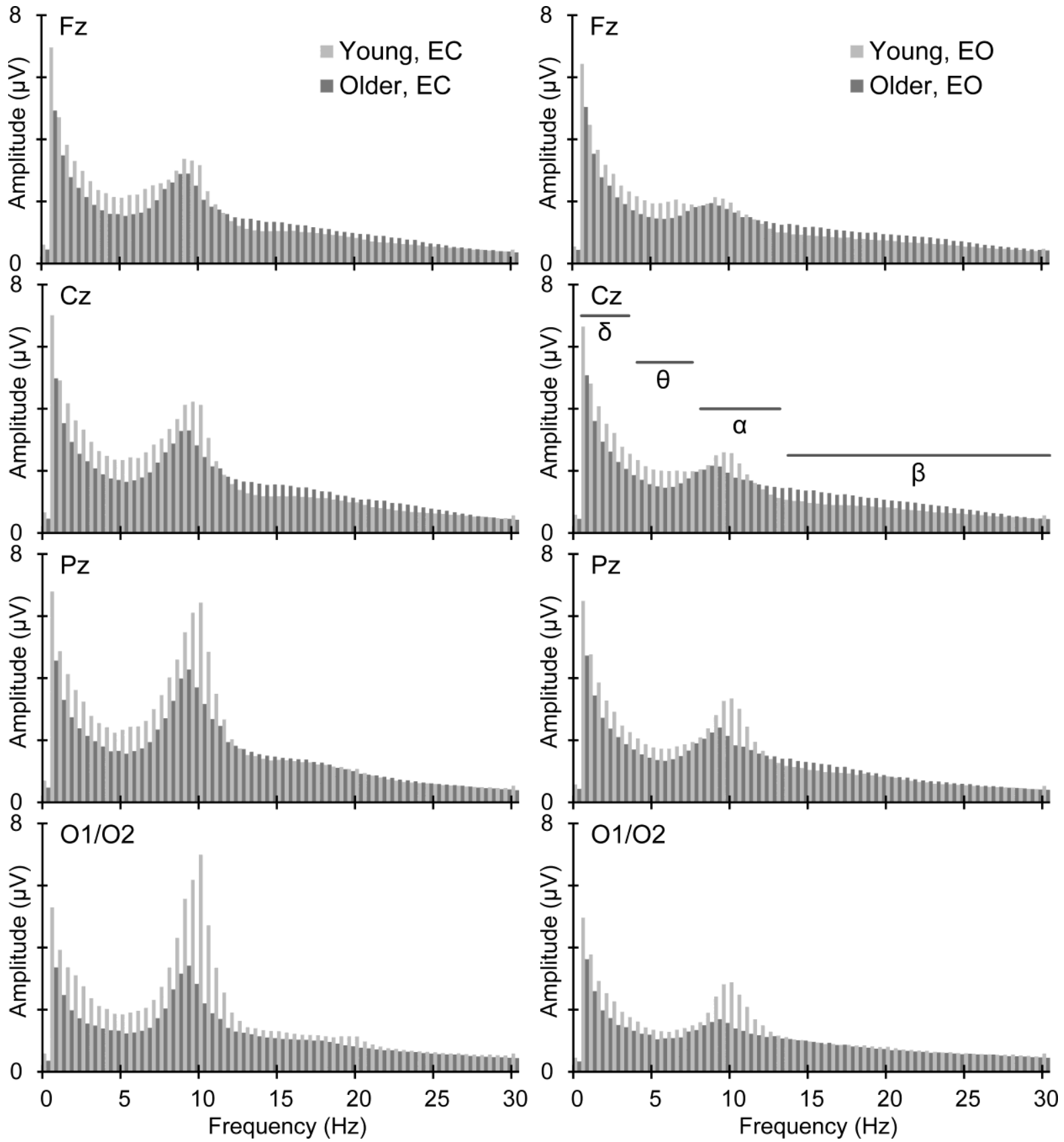

Fig. 1. Grand mean amplitude spectra for EC (left) and EO (right), illustrated at the midline sites and the occipital hemispheric mean, for the Young and Older groups. Traditional frequency band ranges are indicated in the EO plot at $\mathrm{Cz}$.

\subsection{Young f-PCA component outcomes}

The f-PCA of the Young EC spectra yielded nine components, each carrying more than $2 \%$ of the variance, and together accounting for $88.1 \%$. The upper section of Fig. 2 shows, in 
frequency order, the scaled factor loadings and topographic headmaps of these Young eyesclosed (Yc) components, illustrating one delta component (YcD1), a broad delta-theta-alpha component (YcDTA), six alpha components (YcA1 to YcA6) and one beta component (YcB1). For each component, the factor order, variance carried, and peak frequency, are also indicated.

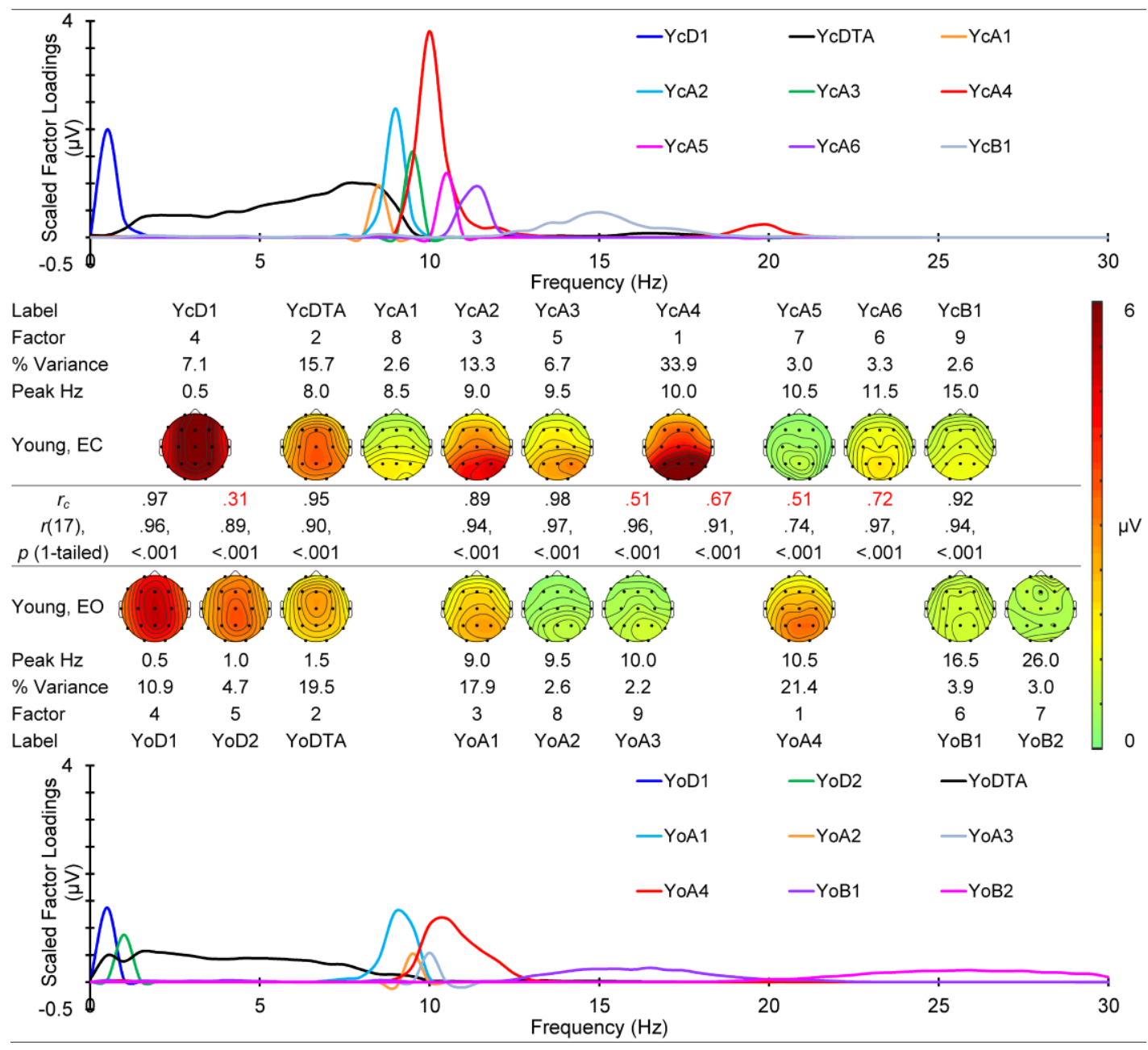

Fig. 2. Young f-PCA outcomes for EC (top) and EO (bottom). The scaled above-threshold factor loadings for each f-PCA are shown as a function of frequency, together with the topographic headmap of each component, variance accounted for, and peak frequency. These are plotted in factor order - i.e., line colour indicates importance in terms of $\%$ variance (e.g., red is the most important). Between the two sets are the Congruence Coefficient $r_{c}$, with coefficients denoting non-similarity indicated in red font, and also the topographic correlation $r(17)$ and its probability between potential component pairs. This figure is available in colour on the web. 
The lower section of Fig. 2 shows the corresponding f-PCA Young eyes-open (Yo) components. This PCA again produced nine components carrying more than $2 \%$ of the variance each, and together accounting for $85.9 \%$. Here there were two delta components (YoD1, YoD2), and a broad delta-theta-alpha component (YoDTA) with greater delta and theta relative amplitude than the DTA of the EC condition (compare scaled factor loadings). There were also four alpha components (YoA1 to YoA4) and two in the beta range (YoB1, YoB2).

Between the upper and lower sections of Fig. 2, the Congruence Coefficient $\left(r_{c}\right)$, and the topographic correlation coefficient $r(17)$ and its 1-tailed probability $p$, are shown for potentially-matching components in the two conditions. YcD1 appears to have split into YoD1 and YoD2, but $r_{c}$ between YcD1 and YoD2 indicates no similarity. Despite their apparent difference in peak frequency, YcDTA matched YoDTA, with $r_{c}$ indicating strong similarity, and with a high topographic correlation. YcA1 at $8.5 \mathrm{~Hz}$ had no frequency match in EO. YcA2 and YcA3 had good matches to YoA1 and YoA2, respectively, at the same frequencies. The remaining Yc alpha components had poor congruence with Yo components. YcB1 matched YoB1 well, despite their peak frequency difference; YoB2 had no EC match. Overall, this initial screening suggested that five Yc components had good matches to five Yo components, despite some differences in peak frequency from EC to EO.

\subsection{Older f-PCA component outcomes}

The f-PCA of the Older eyes-closed spectra also yielded nine components (labelled Oc), each carrying more than $2 \%$ of the variance, and these accounted for a total of $85.9 \%$. Fig. 3 (upper section) shows, in frequency order, the scaled factor loadings and the component topographic headmaps. There were two delta components (OcD1, OcD2), one theta-alpha component (OcTA), four alpha components (OcA1 to OcA4), one broad alphabeta component $(\mathrm{OcAB})$, and one beta component $(\mathrm{OcB} 1)$. The factor order, variance carried, and peak frequency information are also indicated for each component. 


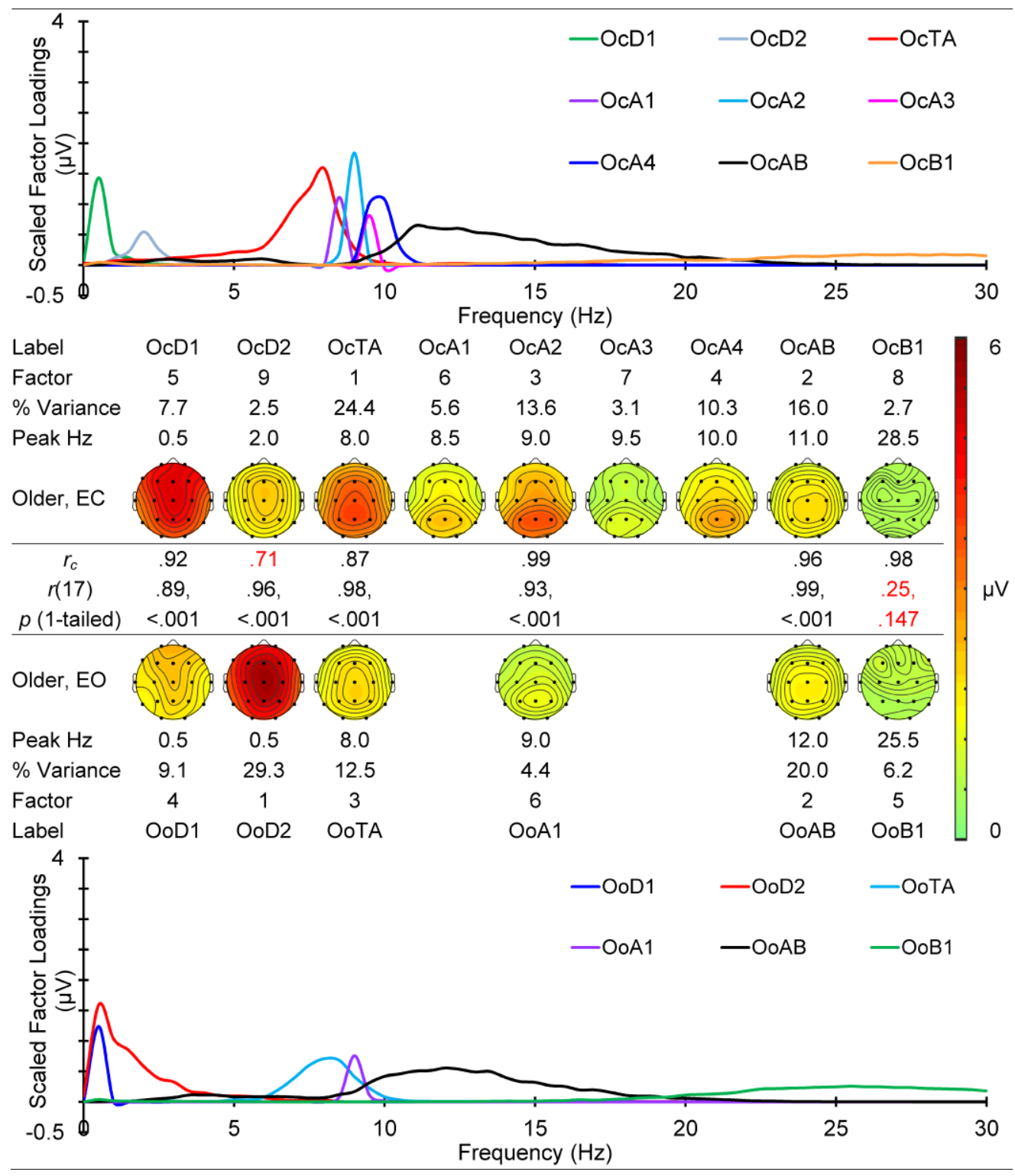

Fig. 3. Older f-PCA outcomes for EC (top) and EO (bottom). The scaled above-threshold factor loadings for each f-PCA are plotted against frequency, and shown with the topographic headmap of each component, their factor order, variance carried, and peak frequency. These are plotted in factor order - i.e., line colour indicates importance in terms of \% variance (e.g., red is the most important). Between the two sets of headmaps, the Congruence Coefficient $r_{c}$, together with the topographic correlation $r(17)$ and its probability, are displayed between potential component pairs. Non-similar $\left(r_{c}\right)$ and non-significant $(r[17])$ values are indicated in red font. This figure is available in colour on the web. 
The lower section of Fig. 3 shows the corresponding results from the Older EO f-PCA. This produced six components (labelled Oo) carrying more than $2 \%$ of the variance each, and together accounting for $81.5 \%$. Here there were two delta components (OoD1, OoD2), one theta-alpha component (OoTA), one alpha component (OoA1), one alpha-beta component $(\mathrm{OoAB})$, and one beta component (OoB1).

Between the upper and lower sections of Fig. 3, the Congruence Coefficient $\left(r_{c}\right)$, and the topographic correlation coefficient $r(17)$ and its 1-tailed probability $p$, are shown for potentially-matching Older adult components in the EC/EO conditions. OcD1 showed a good match to OoD1, and OcD2 showed strong topographic similarity but poor $r_{c}$ with OoD2. OcTA and OoTA at $8.0 \mathrm{~Hz}$ were quite comparable. OcA1, OcA3, and OcA4 had no clear EO match. OcA2 and OoA1 at $9.0 \mathrm{~Hz}$ were equivalent. OcAB was a good match to OoAB, despite the $1 \mathrm{~Hz}$ shift in peak frequency. OcB1 was spectrally equivalent to OoB1, but did not match in topography. Overall, five Oc components seemed to be spectrally matched to five Oo components in the initial screening, despite some differences in peak frequencies, and a topographic shift in beta, from EC to EO.

\subsection{Comparability between components in Young vs. Older Groups}

In a similar approach, the components within each of the Young and Older groups were further screened, first for EC, then for EO. These used $r_{c}$ calculated between the Young and Older unscaled factor loadings, and the correlation $r(17)$ between Young and Older topographies, within each condition. These comparisons indicated that six components in EC were robust across Group, with three components in EO robust across Group. These nine components are brought together in terms of their similarities and statistically compared in the following three sections.

\subsection{Components stable over Conditions and Groups}

Two components robust across Condition (sections 3.2 and 3.3) were also robust across 
Group; one of these components peaked in delta, and one in alpha. Fig. 4 compares each of these in terms of Young versus Older EC headmaps above the corresponding EO headmaps. Between the EC and EO topographies are shown the Congruence Coefficient above the topographic correlation and its associated $p$ value from Fig. 2 (Young) and Fig. 3 (Older); between the Young and Older headmaps are the corresponding data from the Group comparisons (section 3.4).

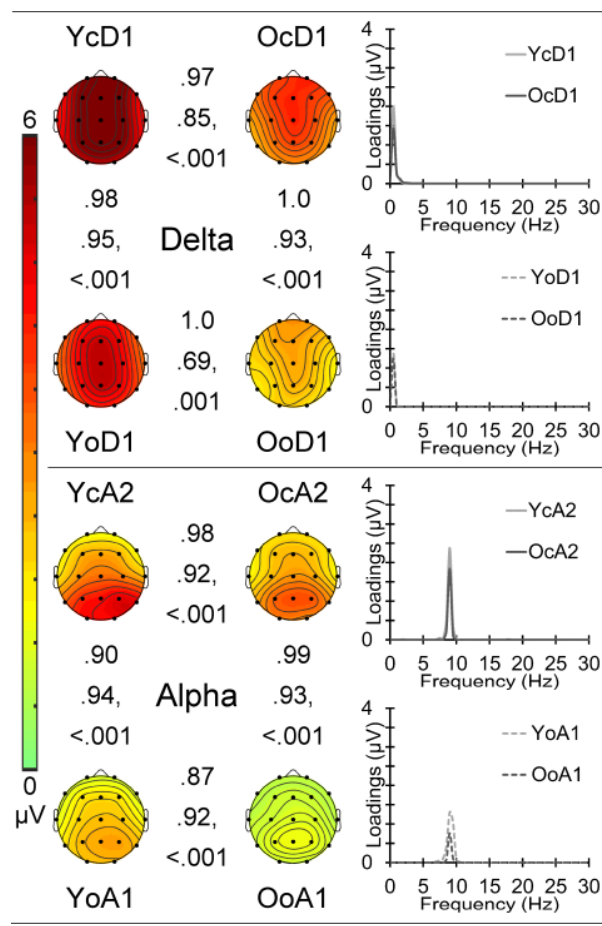

Fig. 4. Delta (upper panel) and alpha (lower panel) f-PCA components that corresponded across Condition and Group, and were assessed in Analysis 1. Topographies are shown on the left, with rescaled factor loadings on the right. Between each pair of headmaps are the Congruence Coefficient $\left(r_{c}\right)$ and topographic correlation statistics $(r$, p) for each match. Note that the headmaps and within-group correspondence statistics are replicated from Fig. 2 (Young) and Fig. 3 (Older). This figure is available in colour on the web. 
Table 1. Statistical outcomes for each analysis.

\begin{tabular}{|c|c|c|c|c|c|c|}
\hline Analysis & Component & Region & Effect & $F$ & $p$ & $\eta_{\mathrm{p}}^{2}$ \\
\hline \multirow[t]{6}{*}{1} & Delta & $\mathrm{Fz}, \mathrm{Cz}, \mathrm{Pz}$ & $\mathrm{EC}>\mathrm{EO}$ & 449.12 & $<.001$ & .92 \\
\hline & & & Young $>$ Older & 60.16 & $<.001$ & .61 \\
\hline & & & Young $>$ Older $\times \mathrm{EC}<\mathrm{EO}$ & 15.98 & $<.001$ & .30 \\
\hline & Alpha & $\mathrm{P} 3, \mathrm{Pz}, \mathrm{P} 4$ & $\mathrm{EC}>\mathrm{EO}$ & 59.51 & $<.001$ & .61 \\
\hline & & & Young $>$ Older & 3.00 & .091 & .07 \\
\hline & & & Young $\sim$ Older $\times \mathrm{EC}>\mathrm{EO}$ & 0.34 & .564 & .01 \\
\hline \multirow[t]{6}{*}{2} & YcDTA, YoDTA & $\mathrm{Fz}, \mathrm{Cz}, \mathrm{P} 3, \mathrm{Pz}, \mathrm{P} 4$ & $\mathrm{EC}>\mathrm{EO}$ & 38.22 & $<.001$ & .67 \\
\hline & YcA3, YoA2 & $\mathrm{P} 3, \mathrm{Pz}, \mathrm{P} 4$ & $\mathrm{EC}>\mathrm{EO}$ & 29.48 & $<.001$ & .61 \\
\hline & YcB1, YoB1 & $\mathrm{Cz}, \mathrm{P} 3, \mathrm{Pz}, \mathrm{P} 4$ & $\mathrm{EC}>\mathrm{EO}$ & 37.73 & $<.001$ & .67 \\
\hline & OcTA, OoTA & $\mathrm{Fz}, \mathrm{Cz}, \mathrm{P} 3, \mathrm{Pz}, \mathrm{P} 4$ & $\mathrm{EC}>\mathrm{EO}$ & 34.08 & $<.001$ & .64 \\
\hline & OcAB, OoAB & $\mathrm{C} 3, \mathrm{Cz}, \mathrm{C} 4, \mathrm{P} 3, \mathrm{Pz}, \mathrm{P} 4$ & $\mathrm{EC}>\mathrm{EO}$ & 17.77 & $<.001$ & .48 \\
\hline & OcB1, OoB1 & All [19] sites & $\mathrm{EC}<\mathrm{EO}$ & 80.35 & $<.001$ & .81 \\
\hline \multirow[t]{4}{*}{3} & YcDTA, OcTA & $\mathrm{Fz}, \mathrm{Cz}, \mathrm{P} 3, \mathrm{Pz}, \mathrm{P} 4$ & Young Older & 0.19 & .668 & .00 \\
\hline & YcA1, OcA1 & $\mathrm{P} 3, \mathrm{Pz}, \mathrm{P} 4$ & Young $\sim$ Older & 1.67 & .203 & .04 \\
\hline & YcA3, OcA3 & $\mathrm{P} 3, \mathrm{Pz}, \mathrm{P} 4$ & Young $>$ Older & 11.37 & .002 & .23 \\
\hline & YoB2, OoB1 & All [19] sites & Young $\sim$ Older & 1.60 & .213 & .04 \\
\hline
\end{tabular}


The Analysis 1 Condition $\times$ Group MANOVA results are shown in the upper section of

Table 1, along with the electrodes contributing to the regional means that were analysed. The delta component, occurring as the first delta component (D1) in each f-PCA, was assessed as a midline component as suggested in Fig. 4. Midline amplitudes in this component showed a significant reduction from EC to EO (across-groups), were larger in the Young than the Older group (across-conditions), and this age-related reduction was greater in EO compared to EC; refer to Table 1 for the relevant statistics.

The stable alpha component occurred as the second alpha component (A2) in each of the EC f-PCAs (Young, Older), and as the first alpha component (A1) in the EO f-PCAs; its different labels are shown in the lower section of Fig. 4. This was assessed as a parietal component, and showed a significant reduction from EC to EO (across-groups), and was somewhat larger in the Young than the Older group (across-conditions), but this did not reach significance; refer to the corresponding Table 1 statistics. The alpha reduction with opening of the eyes did not differ between the Young and Older groups.

\subsection{Components stable over Conditions in only one Group}

In the Young group, section 3.2 indicated three other components that were stable across Condition: one broad DTA component, one alpha, and one beta component. These are shown in the top three rows of Fig. 5, with EC headmaps on the left of the EO headmaps, along with their correspondence statistics (replicated from Fig. 2). The Analysis 2 repeatedmeasures MANOVA results are again shown in Table 1, including the sites used to calculate the component amplitudes, and it can be seen that the relevant regional amplitudes in all three Young components decreased significantly from EC to EO. 


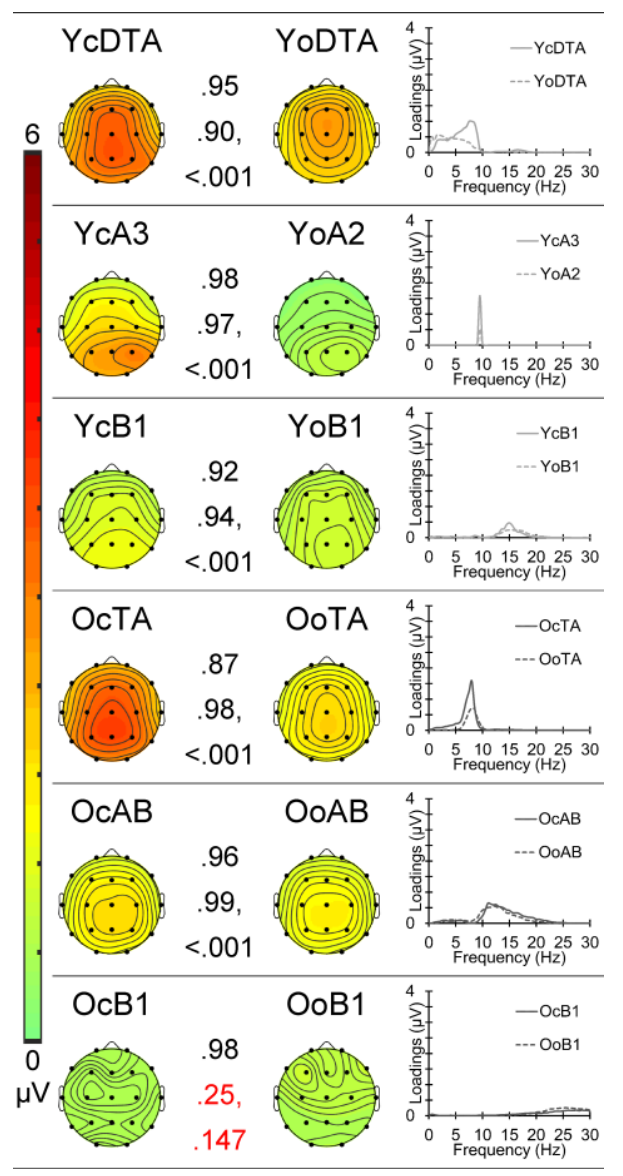

Fig. 5. Component pairs assessed in Analysis 2; these corresponded between conditions, but not between groups. Topographies are shown on the left, with rescaled factor loadings on the right. Reconfigured from Fig. 2 (Young) and Fig. 3 (Older), the EC component headmaps are shown on the left of the corresponding EO components; between each pair are the statistics for their match. The three matching pairs of the Young group are shown above the three different pairs that matched within the Older group. This figure is available in colour on the web.

In the Older group, section 3.3 indicated three other components that were stable across Condition: one theta-alpha component, one alpha-beta component, and one beta component. These are shown in the bottom three rows of Fig. 5, including their $r_{c}$ and the $r(17)$ with its associated $p$ value (reconfigured from Fig. 3). As seen in the relevant Analysis 2 statistics in Table 1, the Older adult theta-alpha and alpha-beta components decreased significantly from EC to EO, but the beta component increased significantly. 


\subsection{Components comparable over Groups in one Condition}

Note that some components assessed in section 3.6 (YcDTA, YcA3, OcTA, OoB1) are assessed here in comparison with a different component. Section 3.4 indicated four Young components, three EC and one EO, that had comparable Older components: a Young EC delta-theta-alpha that matched to an Older theta-alpha, two other EC components in alpha, and one EO component in beta. These are shown in Fig. 6, with component headmaps shown on the left for the Young, and the right for the Older adults; between are the $r_{c}$ and the $r(17)$ with its associated $\mathrm{p}$ value from the component screening conducted in section 3.4. The Analysis 3 between-groups MANOVA results for these components are shown in the lower section of Table 1, together with the electrode sites used to compute their component amplitudes. The only significant group difference in these components was that YcA3 was of greater amplitude than OcA3.

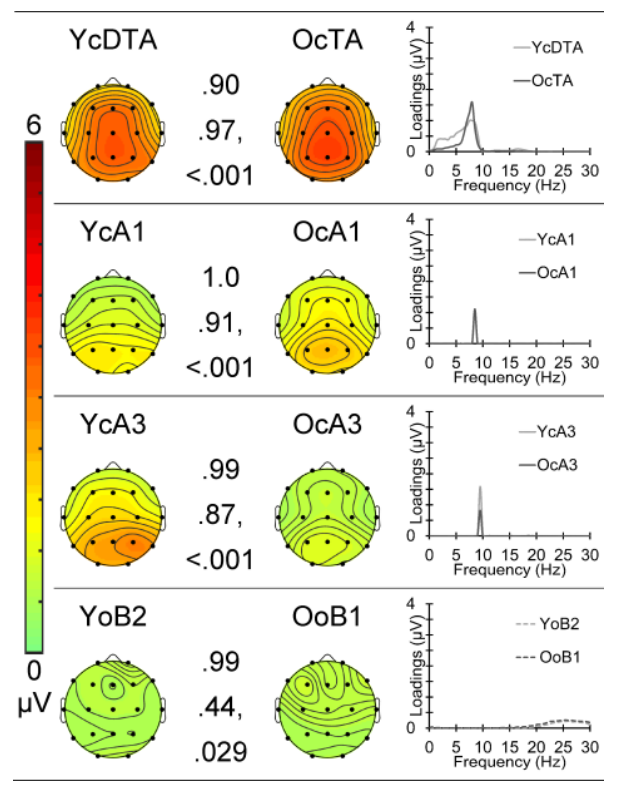

Fig. 6. Component pairs assessed in Analysis 3; these corresponded between groups, but not between conditions. Topographies are shown on the left, with rescaled factor loadings on the right. Component headmaps are shown on the left for the Young (replicated from Fig. 2) with those for the Older group (replicated from Fig. 3) to their right; the $r_{c}$ and the $r(17)$ with its associated $p$ value are shown between each corresponding pair. This figure is available in colour on the web. 


\subsection{Components unique to a single Group and Condition}

There were nine unmatched components apparent from both our within-group and between-group screenings based on $r_{c}$; six in the Young, and three in the Older adults. As these components were unique to a single group and condition, they were necessarily omitted from the formal analyses detailed in Table 1. These included three Young EC alphas (YcA4, YcA5, YcA6), and, in the Young EO decomposition, one delta (YoD2) and two alpha components (YoA3, YoA4), shown in Fig. 2 with red $r_{c}$ values indicating component dissimilarity. In the Older results there was one unique delta component each in EC (OcD2) and EO (OoD2), despite their topographic similarity, and one EC alpha component (OcA4) that had no match.

\section{Discussion}

The f-PCAs of the Young group yielded a sharp low delta component, several alpha components, and one or two beta components in each condition, as expected from previous fPCAs of resting EEG in young adults (Barry and De Blasio, 2018; Barry et al., submitted; Debener et al., 2000; Karamacoska et al., submitted; Kayser et al., 2000; Rodriguez Martinez et al., 2012; Tenke and Kayser, 2005). The novel decomposition of the Older resting EEG spectra generally yielded results comparable with the Young frequency components: a sharp low delta, several alphas, and a beta component. While these separate components appear to be a simple representation of natural rhythms in the traditional bands, there have generally been no discrete theta components reported. Rather, broader delta-theta components, as found here, have sometimes been noted (e.g., Barry et al., submitted; Rodríguez Martínez et al., 2012). Also, other broad components crossed traditional band limits (e.g., the DTA components noted here in both Young and Older, and the AB component noted in the Older group). At present it is unclear whether these are genuine oscillations with frequencies spanning the traditional bands or a reflection of poor component separation in the f-PCAs. This interesting issue, potentially opening new perspectives on the genesis and functionality 
of EEG oscillations, may only be resolved by the accumulation of studies attempting to decompose EEG spectra with a range of methodologies - here we must remain open-minded.

It should be noted, however, that the low delta component, at the limit of resolution of the DFT $(0.5 \mathrm{~Hz})$ may, to an unknown extent, reflect the $1 / \mathrm{f}$ distribution (i.e., amplitude increase as frequency decreases) that is the major feature of the overall EEG spectrum (Bédard et al., 2006; Demanuele et al., 2007), and upon which the alpha band appears to float (see Fig. 1). It is unclear whether this should be regarded as neuronal noise or a genuine EEG oscillation, but it is sometimes excluded in traditional EEG band ranges. However, Barry and De Blasio (2017) included it in their broad delta band, and for comparability, we will do so here. A related point is whether this component is substantially affected by the inclusion of the amplitude value at $0 \mathrm{~Hz}$ in the f-PCA. This question is briefly addressed in the Supplementary Material S2, where we show that this aspect of the f-PCA input has minimal impact on the f-PCA output.

In general, the alpha components noted in both Young and Older groups were parietal in topography and reduced in amplitude from EC to EO - defining characteristics of alpha that generate confidence in those labels.

Our optimised PCA strategy, using separate f-PCAs to avoid misallocation of variance between Condition and/or Group - which would have smeared component frequencies and amplitudes - resulted in a plethora of components. Hence we screened the components for comparability using the Congruence Coefficient (which emphasises similarity of factor loading frequency and amplitude) and topographic correlation (which emphasises similarity of component topographies), and examined effects in the four groupings of components we obtained.

\subsection{Components stable over Conditions and Groups}

The sharp low delta component discussed above had a midline topography and occurred in both Young and Older groups in both EC and EO conditions. Fig. 4 suggests that 
this component was more frontal in the Older group. It was reduced in the EO (cf. EC) condition, and in the Older (cf. Young) group. These Group and Condition effects were comparable with those reported in the traditional delta band investigation of this dataset (Barry and De Blasio, 2017), and the age-related amplitude reduction is highly consistent with the wider ageing literature using traditional EEG-band definitions. The age-related reduction in this natural delta component was greater in EO than EC, compatible with Barry and De Blasio's (2017) report of a central reduction that was also greater in this condition.

One alpha component was found to be compatible between the groups in resting conditions (lower Fig. 4). The parietal amplitude of this component was slightly smaller in the Older than Young group, although this did not reach significance, and there was significantly less amplitude in EO than EC (across-Group); these outcomes are consistent with the traditional alpha band findings in Barry and De Blasio (2017). Interestingly, the EC/EO reactivity did not differ between the groups in this alpha component, a finding that is at odds with the traditional alpha band results of both Barry and De Blasio (2017) and the wider ageing EEG literature (e.g., Duffy et al., 1984; Klimesch, 1999; Könönen and Partanen, 1993).

Together, these results indicate that significant aspects of the traditional band measures of delta and alpha can each be found in a substantial f-PCA component derived independently from the four spectra of EC and EO EEG in Young and Older adults. In these cases, variance associated with topography, EO vs. EC, and age group are each generally reflected in the traditional bands and the natural frequency components.

\subsection{Components stable over Conditions in only one Group}

The Young group had a broad parietal and midline delta-theta-alpha component, a parietal alpha, and a centroparietal beta component that occurred in both EC and EO conditions, but that had no correspondence in the Older group. Each of these was significantly smaller in EO than in EC. In terms of topography and condition effect, the 
delta-theta-alpha component is similar to the Young group's traditional theta band result of Barry and De Blasio (2017), and similar links can also be made between the natural alpha and beta components and the outcomes in the corresponding traditional band data.

The Older group had a parietal and midline theta-alpha component, a centroparietal alpha-beta component, and a diffuse beta component that were found in both EC and EO conditions, with no corresponding components in the Young group. Both the theta-alpha and the alpha-beta components were larger in EC than EO, but the beta component was smaller in EC. The topography and condition effects in this theta-alpha component are similar to the Older traditional theta and alpha band results of Barry and De Blasio (2017), and similar links can be made between the alpha-beta component and the corresponding Older outcomes in the traditional beta band data. The beta component may link to some aspects of the traditional beta band, which showed a frontal increase in EO cf. EC in this sample (Barry and De Blasio, 2017).

These results suggest that there are some natural components that reflect the changes from $\mathrm{EC}$ to $\mathrm{EO}$ conditions that appear in the traditional frequency bands, but that are different in Young and Older populations. They also suggest that some band-specific results are associated with frequency components that span beyond that traditional band. If these stable resting state components are confirmed in future studies, they could prove interesting in their relation to age differences in cognitive and affective functioning - such differences in underlying EEG oscillations might provide new insights into functional processing in normal ageing. Barry and De Blasio (2018) showed how specific f-PCA EEG components can be linked to specific t-PCA ERP markers of cognitive processing, and that approach could be advantageous in such an exploration in normal ageing.

\subsection{Components comparable over Groups in one Condition}

In the EC condition, the midline and parietal Young delta-theta-alpha component corresponded with the Older theta-alpha component, and two parietal alpha components 
corresponded between the Young and Older adults; only the higher-frequency alpha component showed the typical age-related reduction, being significantly larger in the Young than the Older group. The complex component topography of the delta-theta-alpha (Young) and theta-alpha (Older) components appear similar to the traditional theta band EC data of Barry and De Blasio (2017), while the alpha components show some similarity to the EC traditional alpha data, particularly the higher-frequency component showing a reduction with age.

In EO, the small diffuse beta component corresponding between the Young and Older groups showed no significant age-related change, and has no obvious links to the published band data. Together with the outcomes in EC, these data indicate a greater similarity in the EEG spectral component structures between Young and Older participants in the EC condition than in the EO condition, and hence may suggest greater age-related spectral shifts in the oscillations associated with EO. Interestingly, only one of the four component pairs differed significantly in amplitude between the groups (being the higher-frequency EC alpha component), and it would be informative to see how this related to differential processing with increasing age. Perhaps the other three component oscillations, similar across age, contribute to the sometimes-surprising continuity of function in normal ageing.

\subsection{Components unique to a single Group and Condition}

The Young group had three alpha components in EC, and one delta and two alpha components in EO that were not matched, while the Older group had one delta and one alpha component in the EC condition, and one delta component in EO, without matches. The unmatched EO $1.0 \mathrm{~Hz}$ delta in the Young group and the EC $2.0 \mathrm{~Hz}$ and EO $0.5 \mathrm{~Hz}$ delta components in the Older group appear to match the delta band data reported by Barry and De Blasio (2017). This topographic similarity with the unmatched Young EO and the Older EC and EO components suggests that at least some of these sharp low delta components are/reflect genuine EEG oscillations rather than just 1/f noise, although this remains to be 
resolved in future research.

Five of the nine unmatched components were in the EC condition (four in the alpha range), and four in the EO condition (two in the alpha range). This could suggest that there are somewhat more EEG sources active in the EC condition, particularly those contributing to the alpha frequency range, which would be compatible with the general larger spectral amplitudes overall in EC shown in Fig. 1. It would be interesting to try to link these unmatched EC components with specific processes known to be largely restricted to that condition - such as the traditional theta band activity in EC associated with mind wandering (Braboszcz and Delorme, 2011; van Son et al., 2019).

\subsection{Summary and conclusion}

f-PCA quantification offers a convenient and data-driven approach to identify and assess natural spectral components $\mathrm{cf}$. the arbitrary broad traditional band divisions. This method can be easily implemented for spectral data using Dien's (2010) freely available ERP PCA (EP) toolkit. Building on from the early implementation and recommendations of Kayser et al. (2000), we recommend minimising misallocation of component variance via the application of separate f-PCAs for each condition and/or group, and employing covariancebased, unrestricted extraction and Promax rotation. Screening of the resultant frequency components, via the Congruence Coefficient (Lorenzo-Seva and ten Berge, 2006; Tucker, 1951) and topographic correlations, can be used to identify matching natural frequency components in each dataset.

We applied this protocol here to re-analyse resting data in healthy ageing. As expected, this resulted in a plethora of natural components that peaked across the traditional bands, and we logically sorted these into four groupings by screening pairs on the basis of their Congruence Coefficient and topographic correlation. The four groupings ranged from components that were stable over Condition (EC, EO) and Group (Young, Older), to those that were found uniquely in one Condition and Group. 
These different groupings substantially extend our perception of the variability of the oscillations in the resting EEG in normal ageing. Some (but not all) of these components demonstrated age- and/or condition-related changes consistent with those typically reported for their broader traditional band counterparts. Moreover, novel insight was gained as multiple components found to peak within a single traditional band (i.e., alpha) did not show consistent effects of age and/or condition; that is, they appeared to be (to some extent) independently related to these factors. This suggests that such oscillatory components may individually reflect differing functionality, an insight that might be masked with traditional quantification collapsing their data into a single arbitrary broad band. This is a great advantage of f-PCA spectral quantification - the fact that we can visualise what is happening within the broader traditional bands, and find specific/natural components that are sensitive to variables such as ageing and/or condition. Ultimately, this might provide new ways to identify and assess clinical and subclinical deviations in spectral data.

The present study used EEG data recorded DC-30 Hz, excluding the gamma range. Future f-PCA studies including these higher frequencies might open new perspectives on their contribution to both normal and atypical natural oscillations in many situations, including the ageing aspects explored here.

In this investigation we demonstrated the advantages of the f-PCA approach using a healthy ageing application, and have shown that this methodology can build on the traditional EEG literature by providing novel insights into functionally meaningful and naturally occurring oscillatory components at a finer resolution. We believe this methodology has the potential to significantly advance EEG research across its breadth of application. For example, given its fine-grained level of quantification, this technique may allow moredetailed exploration of the EEG-based brain dynamics underlying normal and/or atypical functioning, and contribute to investigations of the EEG-ERP linkages, amongst other applications. Although this is the first application of this approach in an ageing context and the novel data thus require replication, the present study supports the utility of f-PCA in the 
quantification of EEG spectra, and provides a procedural recipe that should prove useful in not only ageing studies, but also a range of other applications.

\section{Acknowledgments}

We thank The Illawarra Retirement Trust (IRT) and the management of the IRT Links Seaside for providing access to their facilities and encouraging resident participation, and also the participants without whom this research would not have been possible. 


\section{References}

Babiloni, C., Binetti, G., Cassarino, A., Dal Forno, G., Del Percio, C., Ferreri, F., . . Rossini, P.M., 2006. Sources of cortical rhythms in adults during physiological aging: A multicentric EEG study. Hum. Brain Mapp. 27, 162-172. https://doiorg.ezproxy.uow.edu.au/10.1002/hbm.20175

Barry, R.J., De Blasio, F.M., 2017. EEG differences between eyes-closed and eyes-open resting remain in healthy ageing. Biol. Psychol. 129, 293-304. https://doi.org/10.1016/j.biopsycho.2017.09.010

Barry, R.J., De Blasio, F.M., 2018. EEG frequency-PCA in EEG-ERP dynamics. Psychophysiology 55, e13042. https://doi.org/10.1111/psyp.13042

Barry, R.J., De Blasio, F.M., Fogarty, J.S., Clarke, A.R., submitted. Natural alpha frequency components in resting EEG and their relation to arousal. Psychophysiology.

Barry, R.J., De Blasio, F.M., Fogarty, J.S., Karamacoska, D., 2016. ERP Go/NoGo condition effects are better detected with separate PCAs. Int. J. Psychophysiol. 106, 50-64. https://doi.org/10.1016/j.ijpsycho.2016.06.003

Bédard, C., Kröger, H., Destexhe, A., 2006. Does the 1/f frequency scaling of brain signals reflect self-organized critical states? Phys. Rev. Lett. 97, 118102. https://doi.org/10.1103/PhysRevLett.97.118102

Braboszcz, C., Delorme, A., 2011. Lost in thoughts: Neural markers of low alertness during mind wandering. Neuroimage 54, 3040-3047. https://doi.org/10.1016/j.neuroimage.2010.10.008

Breslau, J., Starr, A., Sicotte, N., Higa, J., Buchsbaum, M.S., 1989. Topographic EEG changes with normal aging and SDAT. Electroencephalogr. Clin. Neurophysiol. 72, 281-289. https://doi.org/10.1016/0013-4694(89)90063-1

Croft, R.J., Barry, R.J., 2000. EOG correction of blinks with saccade coefficients: A test and revision of the aligned-artifact average solution. Clin. Neurophysiol. 111, 444-451. https://doi.org/10.1016/S1388-2457(99)00296-5 
Cummins, T.D.R., Finnigan, S., 2007. Theta power is reduced in healthy cognitive aging. Int. J. Psychophysiol. 66, 10-17. http://dx.doi.org/10.1016/j.ijpsycho.2007.05.008

Debener, S., Kayser, J., Tenke, C.E., Beauducel, A., 2000. Principal component analysis (PCA) as a tool for identifying EEG frequency bands: II. Dissociation of resting alpha asymmetries. Psychophysiology 37, S35.

Demanuele, C., James, C.J., Sonuga-Barke, E.J.S., 2007. Distinguishing low frequency oscillations within the 1/f spectral behaviour of electromagnetic brain signals. Behav. Brain Funct. 3, 62. https://doi.org/10.1186/1744-9081-3-62

Dien, J., 2010. The ERP PCA Toolkit: An open source program for advanced statistical analysis of event-related potential data. J. Neurosci. Methods 187, 138-145. https://doi.org/10.1016/j.jneumeth.2009.12.009

Duffy, F.H., Albert, M.S., McAnulty, G., Garvey, A.J., 1984. Age-related differences in brain electrical activity of healthy subjects. Ann. Neurol. 16, 430-438. https://doi.org/10.1002/ana.410160403

Fan, J.C., Cheung, R.T., Chu, L.W., Fung, P.C.W., Chang, C.Q., Sik, H.H., .. Gao, J.L., 2014. Age-related changes of EEG and its source in resting state. Proceedings of the 19th International Conference on Digital Signal Processing, 797-800. http://dx.doi.org/10.1109/ICDSP.2014.6900774

Gaál, Z.A., Boha, R., Stam, C.J., Molnár, M., 2010. Age-dependent features of EEGreactivity-spectral, complexity, and network characteristics. Neurosci. Lett. 479, 7984. https://doi.org/10.1016/j.neulet.2010.05.037

Giaquinto, S., Nolfe, G., 1986. The EEG in the normal elderly: A contribution to the interpretation of aging and dementia. Electroencephalogr. Clin. Neurophysiol. 63, 540-546. https://doi.org/10.1016/0013-4694(86)90141-0

Karamacoska, D., Barry, R.J., Steiner, G.Z., submitted. Using principal components analysis to examine resting state EEG in relation to task performance. Psychophysiology.

Kayser, J., Tenke, C.E., 2003. Optimizing PCA methodology for ERP component 
identification and measurement: Theoretical rationale and empirical evaluation. Clin. Neurophysiol. 114, 2307-2325. https://doi.org/10.1016/S1388-2457(03)00241-4

Kayser, J., Tenke, C.E., Debener, S., 2000. Principal components analysis (PCA) as a tool for identifying EEG frequency bands: I. Methodological considerations and preliminary findings. Psychophysiology 37, S54-S55.

Kayser, J., Tenke, C.E., Kroppmann, C.J., Alschuler, D.M., Fekri, S., Ben-David, S., ... Bruder, G.E., 2014. Auditory event-related potentials and alpha oscillations in the psychosis prodrome: Neuronal generator patterns during a novelty oddball task. Int. J. Psychophysiol. 91, 104-120. https://doi.org/10.1016/j.ijpsycho.2013.12.003

Klimesch, W., 1999. EEG alpha and theta oscillations reflect cognitive and memory performance: A review and analysis. Brain Res. Rev. 29, 169-195. https://doi.org/10.1016/S0165-0173(98)00056-3

Könönen, M., Partanen, J.V., 1993. Blocking of EEG alpha activity during visual performance in healthy adults: A quantitative study. Electroencephalogr. Clin. Neurophysiol. 87, 164-166. https://doi.org/10.1016/0013-4694(93)90122-C

Lorenzo-Seva, U., ten Berge, J.M.F., 2006. Tucker's congruence coefficient as a meaningful index of factor similarity. Methodology 2,57-64. https://doi.org/10.1027/16142241.2.2.57

Marciani, M.G., Maschio, M., Spanedda, F., Caltagirone, C., Gigli, G.L., Bernardi, G., 1994. Quantitative EEG Eevaluation in normal elderly subjects during mental processes: Age-related changes. Int. J. Neurosci. 76, 131-140. https://doi.org/10.3109/00207459408985998

Polich, J., 1997. EEG and ERP assessment of normal aging. Electroencephalogr. Clin. Neurophysiol. 104, 244-256. https://doi.org/10.1016/S0168-5597(97)96139-6

Rodríguez Martínez, E.I., Barriga-Paulino, C.I., Zapata, M.I., Chinchilla, C., López-Jiménez, A.M., Gómez, C.M., 2012. Narrow band quantitative and multivariate electroencephalogram analysis of peri-adolescent period. BMC Neuroscience 13, 104. 
https://doi.org/10.1186/1471-2202-13-104

Sleimen-Malkoun, R., Perdikis, D., Müller, V., Blanc, J.L., Huys, R., Temprado, J.J., Jirsa, V.K., 2015. Brain dynamics of aging: Multiscale variability of EEG signals at rest and during an auditory oddball task. eNeuro 2, ENEURO.0067-14.2015. https://doi.org/10.1523/ENEURO.0067-14.2015

Tabachnick, B.G., Fidell, L.S., 2013. Using multivariate statistics, sixth ed. Pearson, Boston MA.

Tenke, C. E., Kayser, J., 2005. Reference-free quantification of EEG spectra: Combining current source density (CSD) and frequency principal component analysis (fPCA). Clin. Neurophysiol. 116, 2826-2846. https://doi.org/10.1016/j.clinph.2005.08.007

Tenke, C.E., Kayser, J., Abraham, K., Alvarenga, J.E., Bruder, G.E., 2015. Posterior EEG alpha at rest and during task performance: Comparison of current source density and field potential measures. Int. J. Psychophysiol. 97, 299-309. https://doi.org/10.1016/j.ijpsycho.2015.05.011

Tenke, C.E., Kayser, J., Pechtel, P., Webb, C.A., Dillon, D.G., Goer, F., ... Bruder, G.E., 2017a. Demonstrating test-retest reliability of electrophysiological measures for healthy adults in a multisite study of biomarkers of antidepressant treatment response. Psychophysiology 54, 34-50. https://doi.org/10.1111/psyp.12758

Tenke, C.E., Kayser, J., Svob, C., Miller, L., Alvarenga, J.E., Abraham, K., . . Bruder, G.E., 2017b. Association of posterior EEG alpha with prioritization of religion or spirituality: A replication and extension at 20-year follow-up. Biol. Psychol. 124, 7986. https://doi.org/10.1016/j.biopsycho.2017.01.005

Tucker, L.R., 1951. A method for synthesis of factor analysis studies (Personnel Research Section Report No. 984). Department of the Army, Washington DC.

van Son, D., De Blasio, F.M., Fogarty, J.S., Angelidis, A., Barry, R.J., Putman, P., 2019. Frontal EEG theta/beta ratio during mind wandering episodes. Biol. Psychol. 140, 1927. https://doi.org/10.1016/j.biopsycho.2018.11.003 
Vlahou, E.L., Thurm, F., Kolassa, I.-T., Schlee, W., 2014. Resting-state slow wave power, healthy aging and cognitive performance. Scientific Reports, 4, 5101. http://doi.org/10.1038/srep05101

Vysata, O., Kukal, J., Prochazka, A., Valis, M., 2012. Age-related changes in the energy and spectral composition of EEG. Neurophysiology 44, 63-67. https://doi.org/10.1007/s11062-012-9268-y

Wang, H., McIntosh, A.R., Kovacevic, N., Karachalios, M., Protzner, A.B., 2016. Agerelated multiscale changes in brain signal variability in pre-task versus post-task resting-state EEG. J. Cogn. Neurosci. 28, 971-984. https://doi.org/10.1162/jocn_a_00947 


\section{Supplementary Materials}

Data-driven derivation of natural EEG frequency components:

An optimised example assessing resting EEG in healthy ageing

Robert J. Barry*, Frances M. De Blasio, Diana Karamacoska

Brain \& Behaviour Research Institute and School of Psychology, University of Wollongong, Wollongong, NSW 2522, Australia

\section{S1. Choice of rotation - Varimax vs. Promax}

Although we follow Kayser and Tenke (2003) in choosing Varimax rotation for temporal PCA (tPCA) of ERP data, our experience in frequency PCA (f-PCA) of EEG spectra suggests that such orthogonal rotation is not optimal. When conducting f-PCAs, we consistently observe negative scaled factor loadings to a greater extent in Varimax compared with Promax rotations. With EEG data, a negative amplitude has no meaning other than mathematical noise, and the reduction of that noise is to be preferred. Further, the component structure obtained with Promax is generally simpler, with sharper components that have less overlapping.

This is illustrated here for fPCA of the Young EC data set, one of the datasets assessed in the paper. Figure S1 shows Varimax-derived loadings above the corresponding Promax loadings; both to the same percentage variance cutoff. Loadings are sharper and better separated in the bottom Promax panel, with separation of two additional components.

Further, this is supported by the correlations between components found with Promax. These ranged from $r=-.07$ to $r=.67$, with a mean (calculated using Fisher's z-transform) of $r=0.31$. Forcing such naturally-correlated components into orthogonality may underlie the poor Varimax outcomes shown in the top panel. 


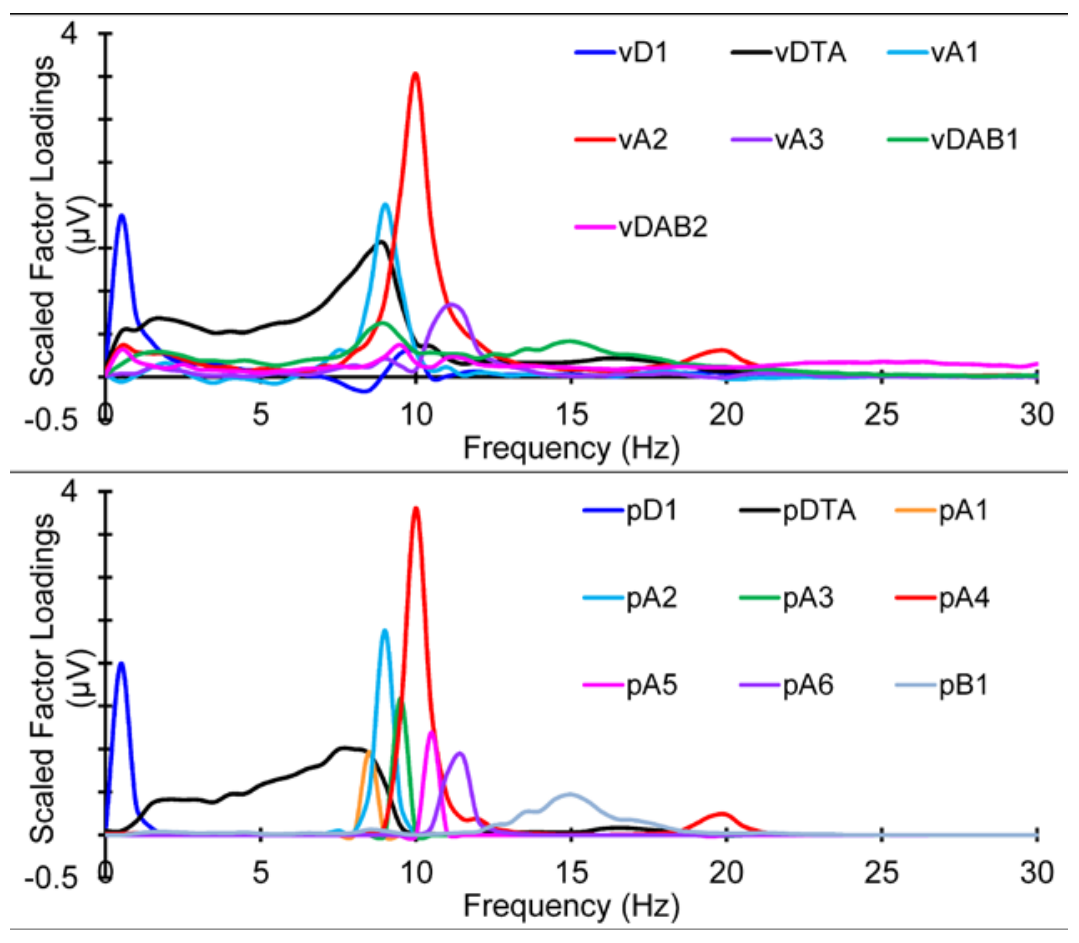

Fig. S1. Scaled factor loadings from the Young EC dataset, obtained using Varimax (top) and Promax (bottom). The Promax set shows little evidence of negative loadings, better simple structure, and some additional components $c f$. the Varimax outcomes. The colouring of the loadings indicates the factor order (reflecting \% variance carried).

\section{S2. Including DC in the spectra?}

An unexplored choice of spectral range input for f-PCA studies is whether to include or exclude the DC component. We explore this issue here.

In our methodology we limit the range of voltage fluctuation in the EEG trace in order to exclude artefact, and this requires that we routinely DC-correct or baseline each epoch. This is done prior to its Fourier analysis to obtain the frequency spectrum. As a result, any spectral activity reported at zero frequency, in our data, arises from the Fourier analysis itself, rather than reflecting recording artefact. Hence we include this DC value as a meaningful amplitude value in subsequent analyses. In the paper, Figure 1 displays the amplitude value at each frequency, including that at $0 \mathrm{~Hz}$.

Not all laboratories follow this procedure. Although not always specified in the methodology, it can be discerned by inspection of their frequency spectra: the spectral distributions from these laboratories do not show an amplitude value at $0 \mathrm{~Hz}$.

The question explored briefly here is whether such an input difference has a substantial impact on the f-PCA component outputs. To this end, we repeated the four f-PCAs of the present study with the amplitudes at $0 \mathrm{~Hz}$ omitted. 


\section{S2.1 Young f-PCA components}

The resultant Young EC and EO data are shown in Figure S2; this corresponds to Figure 2 in the paper. Comparison of Figures 2 and S2 shows a notable difference in that the scaled factor loadings of the EC/EO D1 and EO DTA components here do not begin from baseline at $0 \mathrm{~Hz}$, but rather appear truncated at $0.5 \mathrm{~Hz}$. Apart from this, the two figures and their included data are remarkably similar, with only very minimal changes in variance accounted for (e.g., 7.1 vs. $7.0 \%$ forYcD1) and correspondence statistics (e.g., $r_{c}$ of .97 vs. .98 between $\mathrm{YcD} 1$ and YoD1).

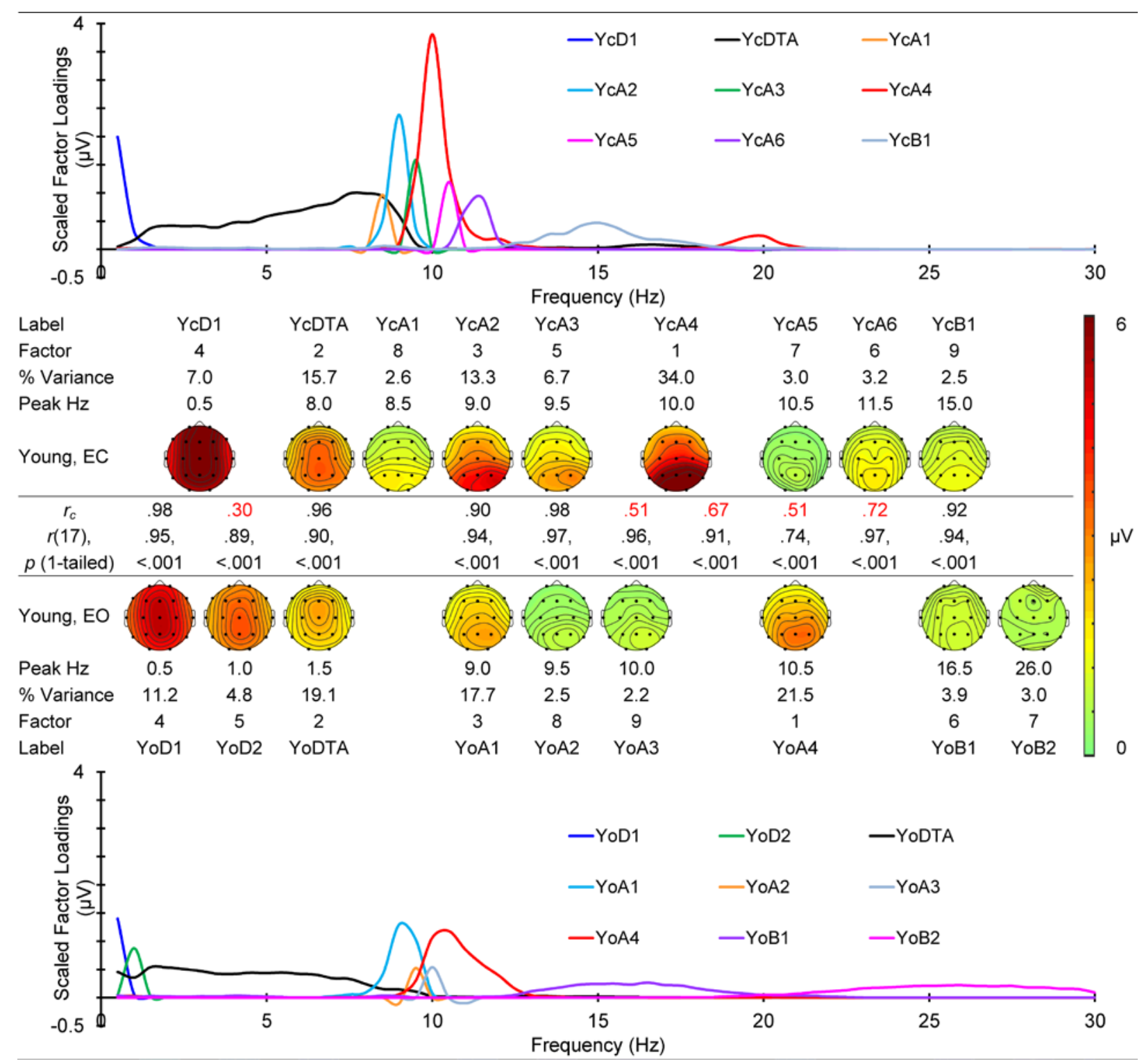

Fig. S2. Young f-PCA outcomes for EC (top) and EO (bottom) derived from frequency spectra omitting $0 \mathrm{~Hz}$ amplitudes; analogous to Figure 2 in the main manuscript.

\subsection{Older f-PCA components}

Figure S3 shows the Older EC and EO f-PCA outputs derived with DC omitted, and corresponds to 
the paper Figure 3. Again, apart from the truncation of the delta component activity at $0.5 \mathrm{~Hz}$ rather than its continuation to $0 \mathrm{~Hz}$, comparison of the two data sets suggests that they are remarkably similar.

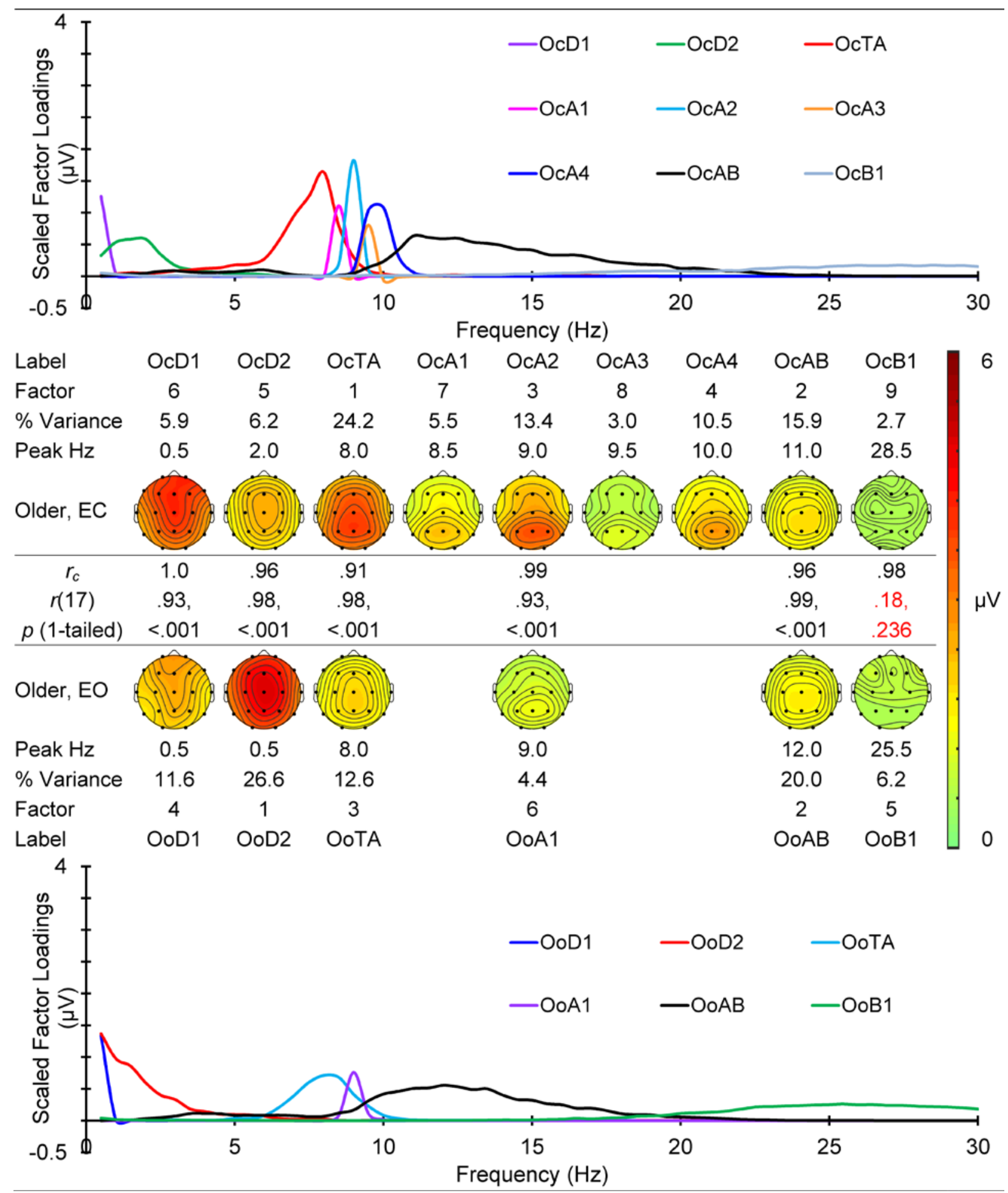

Fig. S3. Older f-PCA outcomes for EC (top) and EO (bottom) after omitting amplitudes at 0 Hz; analogous to Figure 3 in the main manuscript.

\section{S2.3 Conclusion}

The inclusion or exclusion of the amplitudes at $0 \mathrm{~Hz}$ in the input to the f-PCA has little impact on the obtained natural frequency components. 